\title{
Germanica
}

\section{Undine geht ou la dérive vers l'utopie}

Undine geht oder das Abtreiben auf die Utopie hin.

Undine geht or a drift towards utopia.

\section{Martine Sforzin}

\section{OpenEdition}

\section{Journals}

Édition électronique

URL : http://journals.openedition.org/germanica/1053

DOI : 10.4000/germanica.1053

ISSN : 2107-0784

Éditeur

Université de Lille

Édition imprimée

Date de publication : 30 juin 2010

Pagination : 87-106

ISBN : 9782913857254

ISSN : 0984-2632

Référence électronique

Martine Sforzin, « Undine geht ou la dérive vers l'utopie », Germanica [En ligne], 46 | 2010, mis en ligne le 01 juin 2012, consulté le 06 octobre 2020. URL : http://journals.openedition.org/germanica/1053 ; DOI : https://doi.org/10.4000/germanica.1053

Ce document a été généré automatiquement le 6 octobre 2020.

(c) Tous droits réservés 


\title{
Undine geht ou la dérive vers l'utopie
}

\author{
Undine geht oder das Abtreiben auf die Utopie hin. \\ Undine geht or a drift towards utopia.
}

Martine Sforzin

C'est l'ouvrage des dieux :
S'ils ont voulu la mort
pour tant de ces humains,
c'est pour fournir des chants
aux gens de l'avenir.
Odyssée, Chant VIII

Lorsque paraît Die gestundete Zeit en 1953르, Ingeborg Bachmann s'est déjà imposée - elle n'a alors que vingt-six ans - auprès des membres du Groupe 47 comme une voix singulière et remarquable susceptible de faire naître des cendres de l'après-guerre et d'une littérature en ruines une poésie nouvelle ${ }^{2}$. La parution en 1956 du second recueil de poèmes, Die Anrufung des Großen Bären va définitivement révéler à la critique littéraire allemande «la grande dame de la littérature de l'après-guerre $»^{3}$. Mais cette reconnaissance ne satisfait nullement l'intransigeance d'une intellectuelle qui, après des études de philosophie à l'université de Vienne, a réfléchi sur les grands débats philosophiques de son temps : question de l'être et du non-être, métaphysique versus positivisme, philosophie de la vie, linguistic turn et philosophie analytique ${ }^{4}$. L'admiration de ses pairs ne comble pas non plus un auteur perpétuellement en quête d'une esthétique nouvelle, principalement inspirée par le souci du temps présent. Les précurseurs, immenses et écrasants, ont pour nom Trakl, Benn, Brecht ou encore Kafka, Musil, Proust, Joyce, pour se limiter à quelques exemples, sans parler du bouleversement poétiquement fécond et humainement fatal que va représenter la rencontre avec Paul Celan en mai $1948^{5}$. Le respect unanime dont I. Bachmann jouit n'apaise pas davantage un être traumatisé dès l'adolescence par les trahisons d'une patrie aimée, par les forfaitures de l'histoire en marche et les murailles d'incompréhension infranchissables qui en découlent. De même, la conscience aiguë d'une identité de femme fait douloureusement ressentir cette autre frontière qui, 
jusqu'à une échelle individuelle et au plus intime de la vie privée, semble s'obstiner à rejeter l'homme et la femme dans des camps résolument ennemis.

Ainsi ne s'étonnera-t-on pas de cette déclaration en 1963 :

J'ai cessé d'écrire des poèmes lorsque m'a effleurée le soupçon que j'étais désormais « capable » d'écrire des poèmes sans y être contrainte. Et de fait, il n'y aura plus jamais de poèmes tant que je n'aurai pas la conviction qu'il faut que j'en écrive encore qui soient d'un ton si nouveau, qu'ils correspondent véritablement à toute les expériences que j'aurai pu faire entre-temps ${ }^{6}$.

3 De fait, d'autres poèmes suivront et les derniers, composés ou seulement ébauchés entre 1964 et 1967, constituent, selon Hans Höller ${ }^{7}$, la partie la plus importante de l'œuvre poétique d'I. Bachmann', même si la tendance de la recherche depuis la fin des années soixante-dix ${ }^{9}$ est plutôt d'associer la gloire littéraire de la poétesse au projet en prose des Todesarten dont seul Malina est le roman abouti. Mais les propos précédemment cités nous éclairent déjà sur le principe qui préside à l'acte d'écrire chez I. Bachmann et dont elle ne se départira jamais : il ne convient pas d'écrire parce que l'on pourrait céder à la facilité d'une technique acquise et maîtrisée, ou encore pour se prouver que l'on en est capable. Écrire des poèmes n'a de sens que si c'est pour obéir à une nécessité, à un impératif qui échappe au contrôle du libre arbitre ou dépasse des intérêts simplement narcissiques. Le poème ne se justifie plus à ses yeux que si c'est, par la force d'une langue nouvelle, pour dire dans sa totalité la réalité de ce qui a été vécu. Cela revient à prêter à la poésie le pouvoir de traduire en mots cet extrême quasi indicible qui donne à une expérience vécue son caractère unique et authentique et, audelà de toute subjectivité, sa vérité profondément humaine. Or ce pouvoir n'est pas celui, exclusif, de la poésie entendue comme genre littéraire, il doit tout simplement être celui de son matériau, à savoir la langue et les mots, à savoir, encore plus généralement, ce matériau qui fait toute littérature. Dans la deuxième Frankfurter Vorlesung I. Bachmann reprend avec Karl Kraus l'affirmation selon laquelle «toutes les qualités d'une langue s'enracinent dans la morale $»^{10}$. Il pèse ainsi sur la littérature une obligation de vérité, de refus de complaisance et de mensonge. C'est ce même devoir qui fait dire à Kafka, cité au même endroit par I. Bachmann : "Un livre doit être une cognée pour la mer gelée qui est en nous $\aleph^{11}$, avec l'espoir aussi que ce coup de hache libère un tant soit peu les strates d'humanité enfouies tout au fond de l'être - si rarement - humain. C'est dire qu'écrire relève de l'acte éthique. Et I. Bachmann était suffisamment familière de Kafka pour entendre avec lui que l'écriture puisse être une prière. De même, sa connaissance précise du Tractatus a profondément marqué sa pensée et définitivement forgé sa conviction qu'«éthique et esthétique», pour reprendre Wittgenstein, "sont une seule et même chose $»^{12}$.

4 La recherche de la nouveauté qui a pu motiver chez I. Bachmann l'expérimentation de l'écriture en prose, répond ainsi à une volonté éthique, à la nécessité de franchir la frontière du connu et, souvent, du convenu, pour atteindre et découvrir la réalité profonde des situations et des relations humaines et lui ouvrir de nouveaux horizons. Lorsqu'elle aborde dans la première Frankfurter Vorlesung la question de la littérature, des problèmes qu'elle soulève, de ce qu'elle représente, des angles à partir desquels il faut la considérer, I. Bachmann se place dans la perspective de l'écrivain et part du constat que pour qui veut écrire, "le seul maître est l'expérience ${ }^{13}$. Si l'expérience s'entend ici comme la pratique de l'écriture, sa technique, cette dernière ne se ramène nullement à un pur exercice de style ou d'école. Il y va aussi de cette expérimentation avec les possibilités du langage, pour autant qu'elle est susceptible de révéler la vérité 
intime, la vérité de l'expérience. Il y va encore d'un mouvement qui pousse toujours plus loin les limites du langage et qui, de prime abord, nécessite, non de nouvelles techniques, mais, surtout, de nouvelles dispositions d'esprit, une compréhension de l'expérience, une ouverture nouvelle et originale à celle-ci :

Un nouveau langage doit avoir une nouvelle démarche et il n'a cette démarche que lorsqu'il est habité par un nouvel esprit [...] L'écrivain soumet le langage à une transformation et celle-ci ne vise, ni en premier lieu, ni en dernier lieu, une satisfaction esthétique: elle cherche à libérer une faculté nouvelle de compréhension ${ }^{14}$.

5 La conscience aiguë des frontières qui clivent la réalité et la redécoupent en champs hostiles et en camps retranchés appelle un nouveau langage à même de les repousser, de les redessiner, un langage capable d'éroder les frontières qu'il trace lui-même entre les apparences et la réalité, en mesure de ruiner ses propres mystifications ou illusions. Si le récit d'Undine geht retient notre attention, c'est parce qu'il condense la quintessence de l'esthétique d'I. Bachmann en posant la question des frontières à l'intérieur de la réalité, entre la réalité et le langage et parce qu'il illustre de façon performative en quoi un langage nouveau, c'est-à-dire en quoi la recherche du nouveau dans la littérature et dans l'art, illustre cet effort de nature éthique consistant à repousser le plus loin possible les frontières qui enferment dans l'habitude délétère, les discours et les comportements convenus, le faux-semblant et, pour finir, dans l'incompréhension qui divise.

6 Le récit Undine geht vient clore le premier recueil en prose publié en 1961 sous le titre Das Dreißigste Jahr ${ }^{15}$. Les écrits en prose d'I. Bachmann de la fin des années 50 et du début des années 60 sont inscrits dans un présent marqué par le passage d'un mode de penser et de sentir totalitaire et autoritaire à un mode démocratique et pluraliste, marqué, en conséquence, par toutes les répercussions d'une telle transition, qu'il y aille de la conscience politique, du rapport à la Heimat, de la question de l'identité, de la culpabilité dans les crimes nazis, du poids des conventions et de la tradition, de l'appréhension de la réalité et du relativisme, de la crise des métarécits, de la redéfinition des rôles entre les sexes, de la réflexion sur le statut de l'art ${ }^{16}$. Le fait que chaque récit soit plus particulièrement centré sur l'un ou l'autre de ces thèmes tend moins à illustrer la diversité des sujets abordés que leur intrication, leur poids et la place qui leur revient dans une œuvre autoréflexive qui sans cesse s'interroge sur ce qu'est l'art, ce qu'il peut être, ce qu'il se doit d'être. Et Undine geht arrivant en point d'orgue de ce recueil ne manque pas, comme nous le verrons, de soulever cette question, tout en autoréalisant une réponse possible.

7 Le nouveau, chez I. Bachmann, ne surgit pas de rien ou d'un simple mouvement de table rase; il naît en effet d'un long héritage littéraire, culturel, biblique, rigoureusement analysé, repensé et retravaillé. Ainsi le titre du récit charrie-t-il, à lui seul, tel un fleuve au cours ininterrompu, une tradition qui va du mythe à la littérature, qui puise chez Homère pour remonter à Giraudoux en passant par Paracelse, Jacob Böhme et Friedrich de la Motte Fouqué ${ }^{17}$. Le seul nom d'Undine évoque, pêle-mêle, l'idée de séduction, de fascination mais aussi d'abandon, d'infidélité et de trahison, il signale la tension entre désir de vivre et jeu avec la mort; en surimpression se dessinent des catégories aussi opposées que rêve ou mythe et réalité, illusion ou magie et vérité. Cependant, le récit n'illustre pas, comme chez F. de la Motte Fouqué, l'opposition irréductible entre la société humaine, ses règles restrictives et un univers originel où une vision romantique du monde réunit l'homme et la nature, l'homme et la 
femme dans une seule et même âme, une seule et même source ; sans doute se rapproche-t-il davantage du fond tragique de l'ondine de Giraudoux mais le rêve d'amour absolu n'y est pas enterré dans un fossé le séparant à tout jamais d'un monde humain coulé dans le moule du mensonge et de l'artifice. Le lecteur ne retrouve pas ici les oppositions binaires et logiques classiques; le récit reconstitue plutôt, dans une nouvelle complexité, l'écheveau de ces antagonismes, non pour retrouver l'ordre habituel du monde, les frontières habituelles de ses catégories et de ses classifications, mais au contraire pour les contester et faire signe vers un autre ordre possible. Pour ce faire, il brouille d'entrée de jeu les pistes.

\section{Le retournement de la tradition et du mythe}

8 Le titre en partie éponyme met en avant le nom d'Undine, soulignant à la fois l'affirmation de cet être, son identité féminine mais également ce qui se perçoit au premier abord comme sa fragilité, son exclusion du monde, puisqu'il l'annonce «s'en allant » (Undine geht). Or au-delà du titre qui prépare à un récit de type auctorial, ce nom n'apparaît plus une seule fois, un "je » accusateur éclipsant dès les premières lignes le narrateur d'emblée supposé ${ }^{18}$. Ce contraste brutal qui vient surprendre le lecteur, invite d'entrée de jeu à entendre le récit moins comme la narration des avatars d'Undine que comme la capture instantanée d'une attitude et d'un positionnement générés par une expérience limite (celle de l'abandon, de la trahison, de la solitude absolue). Celle-ci est résumée et condensée dans le titre, le texte faisant l'économie de sa remémoration ou, plus précisément, de sa restitution détaillée et de sa description réaliste, pour laisser place à un monologue en forme de rhapsodie qui cristallise souvenirs, regrets, constats, espoirs, anathèmes, objurgations, suppliques. Le texte ne dresse pas à proprement parler un bilan, pas plus qu'il ne tire véritablement une leçon : outre que la structure circulaire du récit suffirait déjà à contredire une telle affirmation $^{19}$, tirer un enseignement supposerait en effet, par rapport à l'expérience faite et à la souffrance qui en a découlé, une distance que le sujet n'est pas entièrement en mesure de prendre. Par contre, le texte vibre de ce qui, derrière la carapace lisse de l'existence, en fait la pulsation intime; il laisse percer le fiel, entendre la colère, le dépit, la nostalgie, il recueille le débordement imprévisible des émotions qui devancent la pensée ; il rend la parole à un «je » dans cet instant créateur qui le pousse à l'excès ou appelle l'ascèse, qui le livre à l'amour sans le délivrer de l'exécration, qui redessine son rapport au monde, détermine si le sujet y trouve sa place, se refuse à l'occuper, en est rejeté ou s'y fait désirer, à quelles conditions et à quel prix. Undine geht est ainsi à considérer sous un double aspect: d'une part, comme la réinterprétation d'une figure féminine à première vue mi-humaine, mi-nature, héritière, certes, de représentations séculaires, mais qui s'en affranchit. En refusant de brider sa liberté et d'altérer son intégrité dans le carcan d'une norme inique, obsolète et criminelle, Undine s'affirme, sous la réécriture d'I. Bachmann, comme un être nouveau qui jette par-dessus bord les chaînes ancestrales, patriarcales. Undine geht est à considérer, d'autre part, comme la figuration d'un être-au-monde qui puise dans le vif des sensations et des émotions, dans l'illimité de son origine (symboliquement aquatique) le refus obstiné de se laisser fossiliser dans le monde aussi ordinaire que féroce des hommes ${ }^{20}$.

Undine n'est pas un être surnaturel, elle ne vit ni au milieu des lutins et des nymphes, ni surveillée par le redoutable Kühleborn, vieil oncle qui règne sur la forêt et se cache 
sous le moindre frémissement de l'onde. Elle est néanmoins un être de nature, c'est de là qu'elle tire son origine. Mais cette origine qu'est pour elle, semble-t-il, l'élément aquatique, ne fait pas tout son être ; l'eau est juste un lieu d'origine, un ancrage à partir duquel Undine part, ou encore, retourne, chez les hommes; il s'agit d'un lieu, point de mire et de fuite à la fois, vers lequel, littéralement désenchantée, elle se réfugie, un lieu qu'elle part retrouver après s'être détournée des hommes. Bien qu'ancrée dans cet élément naturel qu'est l'eau, l'humaine Undine partage avec les hommes cette faculté qui les distingue essentiellement des êtres de nature, à savoir le langage. C'est en effet à travers la parole, à travers un discours, qu'elle se présente à nous et qu'elle existe du début à la fin du récit. Quant à la séparation naturelle et physique d'avec les hommes, elle est loin d'être infranchissable, puisque la clairière qui les éloigne l'un de l'autre les rapproche et les relie tout autant. Depuis l'univers aquatique, ce liquide matriciel et amniotique d'où elle vient, Undine a comme naturellement accès à la clairière, ce que laisse entendre l'image des branches qui s'écartent sur son passage ${ }^{21}$. Au fur et à mesure qu'elle avance, sous le zèle empressé des ramages et des feuilles qui éloignent toute trace d'eau de son corps, sa silhouette humaine se précise. Surtout, le passage dans la clairière semble la rendre à son être véritable mais ce dévoilement se fait au contact d'une nature érotisée: les rameaux métamorphosés en bras accueillants semblent vouloir préparer les siens à l'étreinte, la caresse des feuillages fait sentir sa chevelure. L'anthropomorphisation de ce lieu privilégié ${ }^{22}$ fait comprendre que c'est la puissance de l'amour qui révèle Undine dans la profondeur de son être. Le passage dans la clairière prépare à cet événement qu'est la rencontre avec la vérité profonde de son propre être. Mais la rencontre de soi avec soi n'est possible pour Undine que par l'intercession de l'amour et d'un échange tout aussi à nu avec l'Autre ${ }^{23}$. Ce dévoilement est corrélatif de celui de l'Autre ${ }^{24}$, lequel apparaît bel et bien dans la clairière, et se présente à Undine sous nul autre voile que sa plus simple identité, dans sa singularité, sous son simple nom : «Hans». Ce Hans-là, un parmi les autres, est celui qui s'est momentanément arraché à l'obscurité de la forêt, à l'indifférenciation qu'elle favorise ou encore, pour le dire avec Heidegger, à la domination $d u$ «on $»^{25}$, il est celui qui vient chercher l'éclaircie de son existence, la coïncidence de sa singularité, dans ce qu'elle a d'accidentel et de fragmenté, avec son être, dans ce qu'il a d'absolu et de létal, d'inaltérable, d'authentique. La rencontre entre Undine et Hans semble être le fait du hasard ${ }^{26}$. Espiègle, l'Ondine de Giraudoux déclare à la reine Yseult qui lui demande pourquoi elle a choisi Hans : « Je ne savais pas que l'on choisit, chez les hommes. Chez nous l'on ne choisit pas, de grands sentiments nous choisissent, et le premier ondin venu est pour toujours le seul ondin $\$^{27}$. Ici, la réminiscence fait entendre le sens tragique qu'elle prend avec Undine. Pour elle en effet, le dévoilement de l'être et la possibilité de la rencontre devraient se réaliser comme un destin; le trivial et drôle " on ne choisit pas" d'Ondine devient un appel qui détermine la rencontre dans la clairière et attire Undine et Hans l'un vers l'autre ${ }^{28}$. L'emploi du prétérit, doublé de l'itératif «toujours", ne laisse aucun doute sur la catastrophe, au sens tragique du terme, qui suit la rencontre. L'effort sans cesse renouvelé de réaliser sa destinée, d'être à l'unisson de son être, est régulièrement rendu vain. La moisson de la rencontre est invariablement rendue à l'oubli, lâchement reniée par ce Hans tenté un moment d'accéder à la plénitude de son être mais encore plus vite tenté de redevenir le premier venu, un ondin que rien ne distingue plus des autres (si ce n'est, comme dirait l'Ondine de Giraudoux, des défauts et des maladresses en plus) ; par la faute de l'homme, si l'on en croit le réquisitoire des pages ultérieures, l'événement que constituait la révélation 
de l'être profond de chacun, la renaissance à cet être, est trahi et reste lettre morte. Il n'en reste que l'appel dont Undine seule, repartie dans l'eau du Léthé, réentend toujours l'impérieuse nécessité: "Longtemps après que vos baisers, votre semence auront été emportés au gré des ondes innombrables - pluies, fleuves, mers - le nom demeurera, se propageant sous l'eau, car je ne peux m'empêcher de l'invoquer, Hans, Hans... $»^{29}$.

10 Ni être surnaturel, ni répétition du mythe, Undine est tragiquement humaine, elle ne cherche pas à entraîner les navigateurs de passage dans le naufrage mais la ruse qu'ils déploient pour échapper à leur destin la renvoie à chaque fois à son élément originel. Pour autant, celui-ci n'est pas, comme nous l'avons vu, dissocié de celui des humains ; Undine n'a pas cette in-humanité des sirènes de l'odyssée dont le chant irrésistible ne sert que d'appât pour leur appétit anthropophage.

Comme chez Giraudoux déjà, la force de séduction d'Undine n'a plus rien de démoniaque. Mais Undine n'est pas non plus le simple produit de l'imagination des hommes ${ }^{30}$. Plus qu'une simple projection, elle existe bel et bien, et peut, tout autant, être objet de séduction des hommes que les séduire par son chant ${ }^{31}$. Lorsqu'elle se promet de ne plus répondre à leurs invites, de ne plus se laisser aller à partager les moments privilégiés de leur existence ${ }^{32}$, elle reconnaît s'être laissée attirer par eux ${ }^{33}$. Elle est cet Autre, comme femme et comme humain à la fois, qu'ils ont appelé de leurs vœux $^{34}$; elle représente cette altérité qui leur laisse entrevoir l'apérité et les autres possibles de leur existence. Mais lorsqu'Undine consent à aller vers eux, c'est dans un mouvement de réciprocité qui va rendre possible la rencontre, chacun se livrant et, dans le même instant, livrant bataille. Autrement dit encore, Undine existe bien, tout à la fois altérité objective, et subjectivité qui, à la faveur d'instants de grâce, se découvre autre.

Séduite, Undine n'échappe ni à l'abandon ni à la trahison ${ }^{35}$. Sa séduction à elle n'use pas d'artifices mais bien d'une autre langue avec une autre logique, une langue qui peut être musique, chant ou encore silence. C'est que l'Undine d'I. Bachmann, à la différence du sort que lui réserve la tradition littéraire, est un être affranchi. Elle apparaît et parle au nom d'un « je » qui n'a pas à justifier ou légitimer son identité par la voix des autres ou par une quelconque reconnaissance paternelle ou religieuse ${ }^{36}$. Femme libre et affranchie plus qu'émancipée au sens militant du terme, aucun maître ne lui fera dire, comme à Ondine: "De cette minute je suis la servante de mon seigneur Hans " $^{37}$. Undine dit oui à l'amour, mais non à sa destitution dans l'asservissement de l'épouse, oui à l'enfant, mais non à la femme réduite au rôle de génitrice. Elle refuse moins les liens du mariage ou le projet d'un enfant que leur instrumentalisation par des hommes dominateurs, parjures et mystificateurs, inventant sans cesse des jeux de dupes dans l'espoir de verrouiller l'avenir et d'en rester les maîtres ${ }^{38}$. La charge d'Undine contre une gente masculine prisonnière de comportements phallocrates, convaincue de sa supériorité sur les femmes, dit l'urgence qu'I. Bachmann voyait à s'insurger contre les ravages de la société patriarcale, à dénoncer des modes de pensée assujettis à la loi du père. Pour autant, une partie de la critique féministe a cru déceler, pour le déplorer, que les rôles de victime dans lesquels ses héroïnes apparaissaient, contribuaient, en fin de compte, à les y enfermer et ne faisaient que révéler une soumission à l'ordre patriarcal. Une autre partie s'accorde au contraire à voir dans les héroïnes du recueil Simultan, par exemple, des modèles de femmes qui cherchent à vivre autrement que dans la dépendance sociale et psychologique des hommes. Malina, ou encore Das Buch 
Franza, ne représentent-ils pas, de fait, une tentative de résistance - même si c'est dans le repli vers la folie - à cet ordre patriarcal qui, au cœur de l'ordinaire, génère aussi des criminels et fait le lit du fascisme ? Depuis la fin des années quatre-vingt, une critique poststructuraliste voit précisément dans la prose d'I. Bachmann l'expérimentation d'une esthétique nouvelle qui permet à une parole féminine d'affleurer et de s'arracher au silence auquel la logique masculine la condamnait.

Undine geht nous paraît emblématique d'une œuvre et d'un auteur qui ne prend pas parti pour la cause des femmes sous une forme militante, sans se dérober pour autant aux questions du temps présent et à la responsabilité morale qu'elles soulèvent. La critique de la condition avilissante faite aux femmes semble en effet indissociable pour I. Bachmann de son imbrication dans des rapports de force et des relations complexes s'exerçant à différents niveaux en même temps et fondées sur la violence. Dimensions autobiographique, psychanalytique mais aussi collective, politique et culturelle se mêlent, exacerbant encore davantage les antagonismes entre part féminine et part masculine de tout être. Réfléchir à une émancipation de la femme, c'est, chez I. Bachmann, penser dans un même mouvement ce réseau serré de tensions qui échouent à s'harmoniser et qui est contaminé par ce qu'elle appelle «le virus du crime $»^{39}$; cela revient, même si la perspective adoptée est, le plus souvent, celle d'une femme avilie, blessée et mutilée, voire assassinée ${ }^{40}$, à réfléchir plus globalement à une émancipation de la personne humaine dans le nœud des conflits violents qui la déchirent, sans parler de celui de ses multiples identités, féminine, masculine et androgyne à la fois. Les souffrances que génère la différence des sexes ne sauraient se résoudre, ni dans un rêve - qui s'avérerait vite naï - d'égalité, ni par un retournement radical instaurant en lieu et place de la loi du père un quelconque royaume des Amazones ${ }^{41}$. Le sort dévolu aux femmes, s'il doit être combattu, ne peut l'être en encensant un sexe contre l'autre. Moins prosaïquement, ce combat ne doit pas faire perdre celui de l'amour et des possibilités de liberté qu'il réserve.

Le sort de l'amour est d'être trahi, raison pour laquelle Undine en parle au passé et jure, pour l'avenir, qu'elle ne dira plus jamais oui, plus jamais toi ${ }^{42}$. Mais renoncer au bonheur de l'amour, n'est pas renoncer à l'amour lui-même, à la nécessité de son appel, aux possibles qu'il ouvre. C'est cette obstination qui distingue Undine du commun des Hans qu'elle rencontre. C'est d'elle qu'elle nourrit sa colère et sa révolte. Délaissée, c'est néanmoins elle qui choisit de partir et provoque la séparation, adieu, non à l'amour, mais à sa trahison : «je fis un geste de la main pour signifier la fin. Pour cette fin qui n'en finit pas. Rien n'était jamais fini ${ }^{43}$. Undine ne vient pas vers le monde, elle s'en détourne ; elle ne vient pas y mendier sa place mais sa présence dans la clairière vient faire résonner aux oreilles des Hans aussi multiples que semblales, le vide de leurs existences préformatées. Son appel converge avec celui des hommes, sensibles à « cet appel à en finir $»^{44}$ avec ce qui les sépare d'eux-mêmes et de leurs aspirations profondes. C'est dans ce renversement de perspective que s'opère le retournement du mythe et de la tradition. Et si Undine ne vient pas d'un ailleurs étrange et inquiétant, comme ses lointaines cousines, elle représente bel et bien le lien à un ailleurs qu'il faut à tout prix garder vivant. 


\section{Naufrage du récit et éthique de l'art}

15 En se détournant du monde des humains, Undine affirme sa force, qui n'est autre que sa volonté d'obéir non aux sentiments mais à sa loi, celle-là même qui lui commande de résister aux sirènes de la peur et de la lâcheté, qui rendent les hommes étrangers à euxmêmes, les sépare d'eux-mêmes ${ }^{45}$ et les écarte de leur destinée. Undine est, à travers le cri qui la réveille, cette conscience qui vient rappeler aux hommes ce qu'ils abandonnent, les valeurs auxquelles ils renoncent; elle est aussi, dans un mouvement de réfraction, la conscience qui se désole de cette perte. Undine fait figure de gardienne intraitable des mesquineries et des bassesses que la raison humaine parvient toujours à justifier ; aussi irréductible que la mauvaise conscience, elle prévient que sa mémoire est inhumaine ${ }^{46}$. Mais elle est tout autant la gardienne des possibles et des rêves des hommes, ou encore de ce qu'elle appelle leur esprit, de ce souffle qui leur rend la vie et les arrache à la mort. Les hommes espèrent d'Undine, même s'ils le redoutent, le baiser qui va les transporter, les arracher à l'ordre du connu et les ouvrir à une raison supérieure ${ }^{47}$. Loin du récit de F. de la Motte Fouqué, ce baiser mortel n'est plus celui qui vient punir Huldbrant pour sa trahison; il n'est plus la mise à exécution d'un quelconque jugement divin mais il vient rendre les hommes à une nouvelle vie. Ici encore la tradition est renversée, Undine ne vient plus se glisser parmi les humains pour quémander une âme, c'est elle qui, à l'inverse, vient révéler leur âme aux hommes. La rencontre se fait, de surcroît, dans un mouvement de réciprocité et d'interdépendance, Undine appelant, semble-t-il, ce qui n'aspire qu'à être appelé, réveillé, tiré de son sommeil, arraché à son anéantissement.

Partant d'une réflexion sur le sentiment esthétique, Jean-François Lyotard met en évidence un mouvement de réciprocité et d'interdépendance entre la sensation et le sujet (qu'il appelle encore l'anima) qui l'éprouve: "L'anima», dit-il, "n'existe qu'affectée. La sensation [...] annonce aussi à l'anima qu'elle ne serait pas du tout [...] si rien ne l'affectait. Cette âme n'est que l'éveil d'une affectabilité [...]. Cette âme ne s'affecte pas elle-même, l'autre seul l'affecte, du « dehors ». Exister n'est pas ici le fait d'une conscience visant son corrélat noématique ni celui d'une substance permanente. Exister est être réveillé du néant de la désaffection par un là-bas sensible $»^{48}$. L'expérience qui affecte l'âme dans le texte qui nous occupe est celle de l'amour absolu, thème majeur de la pièce radiophonique Der gute Gott von Manhattan. Mais on ne peut s'empêcher de voir dans la relation d'interdépendance décrite ci-dessus le paradigme du lien intime que le récit Undine geht met au jour entre l'art et l'existence. Interrogée sur la question de savoir s'il fallait lire Undine geht comme une confession, I. Bachmann a donné cette réponse régulièrement citée : « Undine n'est pas une femme, ni non plus un être mais, pour le dire avec Büchner "l'art, ah l'art". Quant à l'auteur, moi en l'espèce, c'est de l'autre côté qu'il faut le chercher, et donc parmi les gens désignés sous le nom de Hans $»^{49}$. Si Undine représente l'art, il s'agit de l'art conçu non comme représentation mais saisi en son instant et dans son mouvement originaires, l'art vu comme une aesthesis qui vient troubler l'esprit ou l'âme qu'il effleure. Undine revendique tout autant son appartenance à l'eau, son lieu originel, qu'à l'air. Elle n'est que mouvement, souffle, pneuma; elle est cette fluctuation libre de tout ancrage, de toute fixation mortifère, toujours ouverte au nouveau et à l'affirmation de la vie :

Je n'ai pas eu besoin de me faire entretenir, pas besoin de serments, pas besoin

d'assurances, mais besoin d'air, de l'air qui souffle la nuit, le long des côtes, le long 
des frontières, pour pouvoir respirer à nouveau et trouver des mots nouveaux, de nouveaux baisers et dire éternellement : oui. Oui ${ }^{50}$. ombres. Mais bien plus que d'apprivoiser la mort, il revient à l'art le devoir de la détourner. Undine déplore que la plupart des Hans acceptent d'être le jouet de la mort en se prêtant au jeu d'une existence fallacieuse, en sacrifiant à des préoccupations utilitaristes. Loin de tirer un enseignement de la mort par lâcheté qu'ils vivent au quotidien, ils s'en font les esclaves dociles et complaisants ${ }^{51}$. Le seul remède contre pareille atrophie ne saurait être un art de la demi-mesure ni même de la mesure, mais un art qui secoue et ébranle les léthargies, plonge le regard dans les abîmes. Capable d'un abandon sans condition, Undine est aussi l'expression du refus le plus intransigeant; si elle goûte à l'extase, solitude et souffrance lui sont plus familières encore. Le lamento d'Undine, lequel mêle accusation et admonestation, se lit comme la mise en œuvre performative d'un art qui se veut ébranlement et, à tout le moins, attente ouverte. Échappant à toute catégorisation de genre (prose, élégie, chant, poème en prose, monologue, drame...), il a pour fonction principale de rendre présent ce qui l'affecte, de rendre à sa présence ce qui manque à l'existence pour qu'elle soit accomplie. On notera également ici que ce n'est point l'amour qui fait échouer l'art à préserver la vie, mais bien plutôt un défaut d'amour, un défaut de courage des divers Hans rencontrés, qui contraignent Undine à s'en aller ; la réitération de son appel fait néanmoins entendre que la nostalgie d'une autre forme d'existence perdure, elle fait signe vers un ailleurs toujours possible.

Cet autre mode d'être-au-monde possible est aux antipodes des règles qui prévalent dans la société occidentale de l'après-guerre dont Undine geht se veut aussi la critique virulente. Il est le négatif qui révèle combien la société travestit son reflet et, avec lui, la réalité qu'elle prétend servir, comprendre ou, comble de l'illusionnisme, améliorer ; il révèle également le travestissement suprême qui dévoie le langage et le dénature en «langage des escrocs ", pour reprendre la formule célèbre du récit Das Dreißigste Jahr. La société érige les différences en autant de raisons de conflits, de guerre, d'exploitation, de laminage de l'individu; à l'opposé, l'art ouvre, derrière l'appel d'Undine, la voie du retour à une forme d'existence libre de tout calcul d'intérêt, de tout utilitarisme, de toute instrumentalisation; il montre le chemin d'une possible réappropriation de soi, d'un dépassement possible des frontières de toute sorte, d'une unité retrouvée avec soi et avec l'Autre. La rencontre avec Undine réalise ce franchissement des frontières génératrices de mort, elle est nuit transfigurée ${ }^{52}$, entéléchie et se dispense de tout discours : «Mais moi, voilà ce que je vous ai enseigné d'un regard » rappelle Undine et commentant les activités humaines, elle poursuit :

Je vous ai dit : dans tout cela c'est la mort qui est à l'œuvre. Et puis : c'est le temps qui sévit. Et en même temps j'ai dit : toi la mort, décampe! Et toi, le temps, arrête ta course ! Voilà ce que je vous ai dit. Et alors, mon bien aimé, tu as parlé d'une 
voix retenue, tu parlais vrai, tu étais sauvé, libre de tout ce qui nous sépare et tu montrais ton esprit affligé, affligé mais grand, qui est pareil à celui de tous les hommes comme toi et dont c'est la nature de n'être dévolu à aucun usage ${ }^{53}$.

vu, cet instant mystique d'accomplissement de soi, qui ne renvoie à aucune transcendance, ne dure pas, il est voué à être trahi parce que l'être qu'il révèle dans sa profondeur et sa totalité existe certes, mais il existe (ek-siste) toujours hors de soi. Seule une expérience telle que celle de l'amour absolu peut le recentrer sur lui-même. L'art, quant à lui, se doit de tenir éveillé cet être jamais totalement éradiqué de l'âme des hommes. Le chant - exaspéré - d'Undine devient, chez I. Bachmann, réenchantement de l'âme, au prix, non du ravissement d'Ulysse mais du départ douloureusement vécu d'Undine et de son exil vers l'eau ; en d'autres termes encore, son chant est enfantement ${ }^{54}$ qui met paradoxalement en relief tout ce qui dissocie les hommes d'avec eux-mêmes, il est création par un «je » qui choisit d'avancer «sans garantie ${ }^{55}$, là où la plupart des hommes prennent des «options» sur l'avenir. Ici comme chez Nietzsche, l'art naît de la douleur, du désespoir, il témoigne du refus, comme il est dit dans la première Frankfurter Vorlesung ${ }^{56}$, de « dormir par peur d'avoir à nous percevoir, nous-mêmes et notre monde ». Analysant la naissance du récit, Maurice Blanchot le fonde sur la ruse d'Ulysse qui lui permet de déjouer la séduction des sirènes et de «jouer sans péril» avec ces puissances toujours soupçonnées d'être menteuses, voire fictives et irréelles :

Les sirènes vaincues par le pouvoir de la technique, [...] Ulysse n'en fut cependant pas quitte. Elles l'attirèrent là où il ne voulait pas tomber et, cachées au sein de l' Odyssée devenue leur tombeau, elles l'engagèrent, lui et bien d'autres dans cette navigation heureuse, malheureuse, qui est celle du récit, le chant non plus immédiat, mais raconté $[. . .]^{57}$.

Le récit qui ramène Ulysse sain et sauf à Ithaque fait place ici à un récit naufragé, à un chant dissonant, un chant de colère qui ramène Undine seule de l'autre côté de la rive, la tient à distance des impostures et des jeux de toute sorte dans lesquels l'existence humaine perd son âme $^{58}$. Le départ d'Undine, indissociable de la réitération sans fin de l'appel ${ }^{59}$, figure ainsi le double mouvement de l'art: un mouvement d'éloignement volontairement consenti par rapport à des formes d'existence qui nient l'unicité, la totalité de leur être, l'ouverture de leurs possibles et, réciproquement, un mouvement d'écho, de renvoi, à cette forme utopique et régulièrement trahie de l'existence que l'art se donne pour tâche de ne jamais perdre de vue et de faire vivre. Loin de la "navigation heureuse, malheureuse", c'est sous la forme de la rupture, dans l'expression véhémente et syncopée de la douleur que le texte tente de ramener l'existence humaine à son port d'attache. Toujours dans la première Frankfurter Vorlesung, I. Bachmann dit à propos du poète qu'il peut "réussir deux choses: représenter, représenter son époque et présenter quelque chose dont le temps n'est pas encore venu $»^{60}$. C'est dans la rupture, dans la discontinuité du discours telles que le figure le récit Undine geht, dans l'écart qu'Undine maintient entre des formes d'existence par provision et l'intuition de leur forme utopique que l'art, retrouvant « le tranchant de la connaissance et l'amertume de la nostalgie ${ }^{61}$ peut arracher l'existence à la mort. «Dans l'art » dit encore I. Bachmann, « on ne progresse pas à l'horizontale, on ne peut qu'y pratiquer des brèches verticales et c'est un processus qu'il faut toujours recommencer $\aleph^{62}$. Que l'utopie ne soit pas un idéal coupé de la réalité et hors de portée, ne signifie pas pour autant qu'elle soit un idéal jamais définitivement réalisable ; elle représente bien plutôt une direction vers laquelle avancer, à charge pour l'art en général et la littérature en particulier de faire signe vers elle, c'est là leur nécessité 
éthique, leur première tâche étant d'éduquer "à une nouvelle perception, à un nouveau sentiment, à une nouvelle conscience $»^{63}$. En s'exilant à nouveau dans son élément, Undine ne fait qu'affirmer sa volonté de croire à l'utopie d'une transformation possible des hommes qu'I. Bachmann désigne encore de la formule « Un jour viendra » 64. Mais reprendre le chemin de l'utopie, tourner le dos aux hommes et à leurs existences tronquées, mutilées et mutilantes, signifie aussi qu'Undine accepte de recréer la distance qui la sépare d'eux. Cette dérive vers l'utopie n'est cependant pas un égarement vers un ailleurs coupé du monde mais une dé-route librement consentie, afin de remettre sur le chemin de leur destinée les navigateurs rendus sourds et aveugles par leurs propres forfaits et leur propres discours. La dérive d'Undine conditionne la possibilité même de leur éveil à ce qu'ils peuvent être d'autre; elle recrée et rend sensible - comme doit tenter de le faire la littérature, notamment par un travail sur la langue ${ }^{65}$ - la distance entre le réel et le possible.

Si la noirceur du cycle des Todesarten a pu donner le sentiment qu'I. Bachmann se départissait de la foi dans cette utopie de la langue particulièrement prégnante dans les Frankfurter Vorlesungen, elle n'infirme nullement la profonde conviction que seule l'éthique donne à l'art sa justification. En réhabilitant le chant de la sirène, I. Bachmann, sans opposer radicalement hommes et femmes dans leur différence sexuelle, fait porter par la voix féminine "le désarmant appel de l'éthicité opposé aux systèmes armés du discours masculin $»^{66}$; elle a, sur le fond d'un siècle barbare, arraché à son silence la voix féminine qui «se lève à la façon d'un cri d'appel, d'une interrogation " parce qu'elle est «la voix du courage du sens opposé à la répétition mécanique du jeu masculin $»^{67}$.

\section{NOTES}

1. Le recueil paraît pour la première fois à l'automne 1953 aux éditions du Frankfurter Verlag dans la collection dirigée alors par Alfred Andersch.

2. Elle reçoit en mai 1953 le prix du Groupe 47, distinction qui fait autorité dans le paysage littéraire de l'immédiat après-guerre et conforte son choix de se consacrer à la littérature.

3. Nous empruntons l'expression à Sara Lennox dans M. Albrecht / D. Göttsche (Hrsg.), Bachmann Handbuch Leben Werk Wirkung, Metzler Verlag, 2002, p. 25.

4. En 1949 I. Bachmann soutient, sous la direction de Viktor Kraft, une thèse qu'elle présente comme une critique de la philosophie existentiale de M. Heidegger, reprochant au philosophe, dans une perspective logico-néopositiviste une semi, pour ne pas dire, pseudo-rationalité qui chercherait de façon hasardeuse à fonder une ontologie à partir des intuitions, des vécus de la conscience ou d'expériences existentielles et en défendant une attitude herméneutique seule à même de penser l'être là où, I. Bachmann le rappelle dans ce travail, L. Wittgenstein préconise de taire ce dont on ne peut parler. La thèse est aisément consultable sous les références suivantes : I. Bachmann, Die kritische Aufnahme der Existentialphilosophie Martin Heideggers, Piper, 1985. Il n'est pas anecdotique de souligner que cette thèse "contre Heidegger" ( $C f$. I. Bachmann dans Wir müssen wahre Sätze finden. Gespräche und Interviews, Serie Piper, 4. Aufl., 1994, p. 137, ouvrage que nous citerons ultérieurement sous l'abréviation « GuI ».) se termine sur l'idée que c'est à l'art et à 
la poésie, bien plus qu'à la philosophie, qu'il revient de décrire les expériences existentielles qui posent la question du sens et du non-sens de l'existence et I. Bachmann de citer le tableau de Goya «Chronos dévorant ses enfants » ainsi que le poème de Baudelaire « Le gouffre » à l'appui de sa démonstration.

Sur les analogies et les divergences entre Heidegger et I. Bachmann, $c f$. Barbara Agnese, Der Engel der Literatur. Zum philosophischen Vermächtnis Ingeborg Bachmanns, Wien, Passagen Verlag, 1996, p. 75-101.

5. Cf. Hans Höller, Ingeborg Bachmann, trad. fr. Miguel Couffon, Actes Sud, 2006, p. 61-67.

Sur le dialogue poétique entre I. Bachmann et P. Celan $c f$. Sigrid Weigel « Le dialogue avec Paul Celan» dans Europe, Ingeborg Bachmann, août-septembre 2003, p. 84-103, cf. Françoise Rétif «Bachmann, Celan et le mythe d'Orphée», ibidem p.104-127, cf. Françoise Rétif "Ondine bohémienne » dans Alain Cozic, Jacques Lajarrige (Dir.), Traversées du miroir, L'Harmattan, 2005, p. 137-144.

Cf. Bertrand Badiou, Hans Höller, Andrea Stoll et al. (Hrsg.), Herzzeit. Ingeborg Bachmann - Paul Celan. Der Briefwechsel, Suhrkamp Verlag, 2008.

6. „Ich habe aufgehört Gedichte zu schreiben, als mir der Verdacht kam, ich „könne“ jetzt Gedichte schreiben, auch wenn der Zwang, welche zu schreiben, ausbliebe. Und es wird eben keine Gedichte mehr geben, eh' ich mich überzeuge, dass es wieder Gedichte sein müssen, so neu, dass sie allem seither Erfahrenen wirklich entsprechen." dans GuI, p. 40.

7. Cf. Hans Höller, Ingeborg Bachmann. Letzte, unveröffentlichte Gedichte. Entwürfe und Fassungen, Suhrkamp Verlag, 1998, p. 7.

8. Figurent parmi ces derniers poèmes les «Vier Gedichte » écrits à Berlin dans la période de profonde dépression qui suit la rupture avec Max Frisch et publiés en novembre 1968 dans le $\mathrm{n}^{\circ} 15$ de la revue Kursbuch : Keine Delikatessen, Enigma, Prag Jänner 64, Böhmen liegt am Meer, (p. 91-95).

9. En 1978 paraît sous le titre Werke et sous la direction de Christine Koschel, Inge von Weidenbaum et Clemens Münster aux éditions Piper l'édition intégrale en 4 volumes de l'œuvre d'I. Bachmann - elle inclut une partie de l'œuvre posthume - cette édition contribue largement à ce déplacement d'intérêt de la poésie vers la prose.

10. „Alle Vorzüge einer Sprache wurzeln in der Moral“ dans I. Bachmann, Werke 4, Essays Reden Vermischte Schriften Anhang Serie Piper, 5. Aufl. 1993, p. 206 (abréviation : W. 4).

11. „Ein Buch muss die Axt sein für das gefrorene Meer in uns“, W. 4 p. 210.

12. Cf. la proposition 6. 421 : «Il est clair que l'éthique ne se laisse pas énoncer. L'éthique est transcendantale. (Éthique et esthétique sont une seule et même chose.) » dans Wittgenstein, Tractatus logico philosophicus, traduction de Gilles-Gaston Grangier, Gallimard, tel, p. 110.

13. „Und doch ist ja die Erfahrung die einzige Lehrmeisterin“, W. 4, p. 184.

14. „Eine neue Sprache muß eine neue Gangart haben, und eine neue Gangart hat sie nur, wenn ein neuer Geist sie bewohnt [...].[Die Sprache] gehorcht einer Veränderung, die weder zuerst noch zuletzt ästhetische Befriedigung will, sondern neue Fassungskraft" W. 4, p. 192 / Traduction française d'Elfie Poulain, «Leçons de Francfort. Problèmes de poésie contemporaine » dans Ingeborg Bachmann, Euvres, Thesaurus, Actes Sud, oct. 2009, p. 658 sq.

15. Cf. Ingeborg Bachmann, Werke 2, Serie Piper 1702, 5. Auflage 1993, p. 84-263 (abréviation: W. 2). Nous citerons ultérieurement le récit Undine geht (p. 253-263 dans W. 2) sous l'abréviation U.g. Traduction française de Marie-Simone Rollin, "Ondine s'en va ", dans La Trentième année (Edition du Seuil, 1964), recueil repris dans Ingeborg Bachmann, Euvres, Thesaurus, Actes Sud, oct. 2009, p. 135 à 257. Sauf indication contraire, les passages cités seront traduits par nos soins.

16. Cf. l'analyse de Jost Schneider dans Handbuch, op. cit., p. 112.

17. $C f$. Ruth Fassbind-Eigenheer, Undine und die nasse Grenze zwischen mir und mir. Ursprung und literarische Bearbeitung eines Wasserfrauenmythos. Von Paracelsus über Friedrich de la Motte Fouqué zu Ingeborg Bachmann, Verlag Hans-Dieter Heinz, Akademischer Verlag Stuttgart, 1994. L'auteur 
émet en outre l'hypothèse d'échanges intenses sur le thème d'Undine entre I. Bachmann et HansWerner Henze, auteur et compositeur du ballet „Undine“ (ibidem p. 126-132).

18. „Ihr Menschen! Ihr Ungeheuer!

Ihr Ungeheuer mit Namen Hans ! Mit diesem Namen, den ich nie vergessen kann.“ (U.g. p. 253).

19. L'appel sur lequel le récit se termine remet en partie en question la décision de s'en aller annoncée dans le titre. Cf. sur cette structure circulaire, Ruth Fassbind-Eigenheer, op. cit., p. 161-164.

20. "Hommes" pris dans les deux sens, d'êtres de sexe masculin et d'êtres humains, identifiés dans le récit sous le nom de Hans. I. Bachmann s'inspire ici de J. Giraudoux qui prénomme Hans le chevalier von Wittenstein zu Wittenstein, tout à la fois individu falot et peu chevaleresque (bête mais beau, comme le lui dit Ondine) et archétype d'une humanité rayonnante de médiocrité. Et lorsque le roi des ondins adjure Ondine de ne pas aller "chez les hommes ", elle se défend en rétorquant qu'elle va « chez un homme », $c f$. J. Giraudoux, Ondine, Paris, Grasset, Le livre de poche 1657, 2009, p. 52.

21. „Immer wenn ich durch die Lichtung kam und die Zweige sich öffneten, wenn die Ruten mir das Wasser von den Armen schlugen, die Blätter mir die Tropfen von den Haaren leckten [...]“ U.g. p. 253 (souligné par nous).

22. Cf. „Die Ruten ... schlugen / die Tropfen ... leckten“ (U.g. p. 253, souligné par nous).

23. La place que prend ici l'amour met en évidence les différences à partir desquelles I. Bachmann se réapproprie le topos heideggerien de la clairière et le repense.

24. „Wir waren vom gleichen Geist“, se souvient Undine (U.g. p. 258). Ce dévoilement s'accomplit par ailleurs dans le silence, au-delà d'un langage qui expose toujours au travestissement et à la trahison. Cf. le discours pour la remise du Prix Anton Wildgans : „Die Sprache ist die Strafe“ (W. 4, p. 297).

25. „[Ich] traf auf einen, der Hans hie“ (U.g. p. 253, souligné par nous).

26. $C f$. note précédente.

27. $C f$. J. Giraudoux, op. cit., p. 84.

28. En dépit du mouvement qui porte Undine vers Hans, l'appel à la fois lancé et entendu qui les rapproche n'est pas ici la stricte réplique de l'appel heideggerien de la conscience morale, à savoir celui qui convoque le soi-même d'un Dasein qui cesse de « se perdre dans la publicité du on et du on-dit et fait la sourde oreille au soi-même propre " (cf. Martin Heidegger, Être et temps, trad. fr. François Vezin, Gallimard, nrf, 1986, p. 327). Une distinction radicale est maintenue dans le récit entre Undine et Hans et c'est dans la tension de cette séparation qu'Undine rappelle à Hans ce qu'il pourrait/devrait être. Elle le fait au demeurant sur le mode du cri, de la protestation véhémente et douloureuse, là où Heidegger avance : «L'appel se passe de toute émission vocale. [...] La conscience morale parle uniquement et constamment sur le mode du silence » (Ibidem, p. 330). Et s'il arrive à Undine de se retrancher derrière le silence, celui-ci, là encore, se distingue du « mode étrange du silence » (Ibid. p. 332) sur lequel l'appel heideggerien parle, et renvoie à une utopie de la langue (Cf. note 65). Pour I. Bachmann, la morale ne tire pas son origine des profondeurs de l'Être, pas plus que l'art ne saurait être la « mise en œuvre de la vérité de l'Être ».

29. „Und wenn eure Küsse und euer Samen von den vielen großen Wassern -Regen, Flüssen, Meeren - längst abgewaschen und fortgeschwemmt sind, dann ist doch der Name noch da, der sich fortpflanzt unter Wasser, weil ich nicht aufhören kann, ihn zu rufen, Hans, Hans..., U.g. p. 253. / Trad. fr. op. cit., p. 252.

30. Auguste, son père adoptif, explique à Hans : «Souvent quand elle a disparu, nous pensons que c'est pour toujours [...] Quand elle est partie, tout d'elle est parti. Quand elle est partie, elle n'est jamais venue. C'est un rêve, Ondine. Il n'y a pas d'Ondine » dans J. Giraudoux, op. cit., p. 42.

31. „Fahren ihren Frauen, ihren Kindern treulich übers Haar, [...] lauschen den Gang hinunter, [...] und nun hören sie es ganz deutlich: Den Schmerzton, den Ruf von weither, die geisterhafte Musik." (U.g. p. 255). 
32. „denn ich werde nicht wiederkommen, euren Winken nicht mehr folgen, keiner Einladung zu einem Glas wein, zu einer Reise, zu einem Theaterbesuch.“ (U.g. p. 253).

33. et ce, en dépit de leurs imperfections : „Ihr Monstren [...] mit den kurzen blassen Nägeln, den zerschürften Nägeln mit schwarzen Rändern [...]“ (U.g. p. 253).

34. „Doch vergeßt nicht, daß ihr mich gerufen habt in die Welt, dass euch geträumt hat von mir, der anderen, dem anderen [...].“ (U.g. p. 260, souligné par nous).

35. „Ihr habt die Altäre rasch aufgerichtet und mich zum Opfer gebracht.“ (U.g. p. 260).

36. $C f$. les parents adoptifs d'Undine qui préfèreraient «Dorothea, das heiße Gottesgabe » à ce nom païen d'Undine, choisi par ses géniteurs et qui n'est accepté que parce qu'il finit par trouver la bénédiction de l'abbé : dans F. de la Motte Fouqué, Undine, Reclam491, p. 15 sq. et cf. « Moi, on m'appelle Ondine », dans J. Giraudoux, op. cit., p. 29.

37. Cf. J. Giraudoux, op. cit., p. 33.

38. „Ihr Ungeheuer mit euren Frauen! [...] Ja, dazu nehmt ihr euch die Frauen auch, damit ihr die Zukunft erhärtet, damit sie Kinder kriegen [...] Ihr Betrüger und ihr Betrogenen. Versucht das nicht mit mir. Mit mir nicht!“ (U.g. p. 256).

39. I. Bachmann use de cette expression, «das Virus Verbrechen », dans l'ébauche d'avantpropos du roman inachevé Der Fall Franza, cf. I. Bachmann Werke 3, p. 341. Cf. encore dans ce même texte : „[Dieses Buch] versucht, mit etwas bekanntzumachen, etwas aufzusuchen, was nicht aus der Welt verschwunden ist. [...]. Ja, ich behaupte und werde nur versuchen einen ersten Beweis zu erbringen, dass noch heute sehr viele Menschen nicht sterben, sondern ermordet werden“.

40. Cf. la phrase orpheline si souvent citée qui clôt le roman Malina : " Es war Mord ».

41. Retournement auquel ne se résume évidemment ni le mouvement féministe depuis sa naissance ni toute la richesse des analyses apportée par les gender studies.

42. „Ich werde nie wiederkommen, nie wieder Ja sagen und Du und Ja“ (U.g. p. 254).

43. „Ich machte mit der Hand ein Zeichen für Ende. Für das Ende, das kein Ende findet. Es war nie zu Ende.“ (U.g. p. 258).

44. „Ruf zum Ende“ (U.g. p. 257).

45. „Das Gebot ist: Sich verlassen, daß Augen den Augen genügen, daß ein Grün genügt, daß das Leichteste genügt. So dem Gesetz gehorchen und keinem Gefühl.“ (U.g. p. 259) /,Nie wart ihr mit euch einverstanden“" (U.g. p. 256).

46. „Mein Gedächtnis ist unmenschlich.“ (U.g. p. 260).

47. Doch vergeßt nicht, daß ihr mich gerufen habt in die Welt, daß euch geträumt hat von mir, der anderen, dem anderen, von eurem Geist und nicht von eurer Gestalt, der Unbekannten, die auf euren Hochzeiten den Klageruf anstimmt, auf nassen Füßen kommt und von deren Kuß ihr zu sterben fürchtet, so wie ihr zu sterben wünscht und nie mehr sterbt: ordnungslos, hingerissen und von höchster Vernunft". (U.g. p. 260).

48. Dans J.-F. Lyotard, Moralités postmodernes, Galilée, 1993, p. 205.

49. „Die Undine ist keine Frau, auch kein Lebewesen, sondern, um es mit Büchner zu sagen, 'die Kunst, ach die Kunst'. Und der Autor, in dem Fall ich, ist auf der anderen Seite zu suchen, also unter denen, die Hans genannt werden“ dans I. Bachmann, GuI, op. cit., p. 46.

50. „Ich habe keinen Unterhalt gebraucht, keine Beteuerung und Versicherung, nur Luft, Nachtluft, Küstenluft, Grenzluft, um wieder Atem holen zu können für neue Worte, neue Küsse, fûr ein unaufhörliches Geständnis: Ja. Ja.“ ( U.g. p. 254).

51. „[Eure Frauen, eure Kinder] haben euch nicht den Tod gelehrt, sondern nur beigebracht kleinweise.“ (U.g. p. 258).

52. Et non pas seulement „Nacht, zum Schlaf mißbraucht“ (U.g. p. 258).

53. „Aber ich habe euch mit einem Blick gelehrt [...] - ich habe euch gesagt: Es ist der Tod darin. Und: Es ist die Zeit daran. Und zugleich: Geh Tod! Und: Steh still, Zeit! Das habe ich euch gesagt. Und du hast geredet, mein Geliebter, mit einer verlangsamten Stimme, vollkommen wahr und gerettet, von allem dazwischen frei, hast deinen traurigen Geist hervorgekehrt, den traurigen, 
großen, der wie der Geist aller Männer ist und von der Art, die zu keinem Gebrauch bestimmt ist.“ (U.g. p. 258/souligné par nous).

54. Il est les enfants qu'Undine dit ne pas avoir des hommes : „Ich habe keine Kinder von euch“ (U.g. p. 254).

55. Cf. I. Bachmann : „Es ist das Wunder des Ich, daß es, wo immer es spricht, lebt; es kann nicht sterben - ob es geschlagen ist oder im Zweifel, ohne Glaubwürdigkeit und verstümmelt - dieses Ich ohne Gewähr." Dans III. Frankfurter Vorlesung, op. cit., p. 237.

56. . - I. Bachmann, I. Frankfurter Vorlesung, op. cit. p. 198. Trad. fr., op. cit. p. 663.

57. Dans Maurice Blanchot, Le livre à venir, Gallimard, idées nrf, 1959, p. 12.

58. „Ach, so gut spielen konnte niemand, ihr Ungeheuer! Alle Spiele habt ihr erfunden, Zahlenspiele und Wortspiele, Traumspiele und Liebesspiele“ (U.g. p. 262).

59. Le récit se termine sur cet appel, appel ambigu qui peut s'entendre aussi bien comme celui d'Undine que comme celui de Hans.

60. . - I. Bachmann, I. Frankfurter Vorlesung, op. cit. p. 196. Trad. fr., op. cit. p. 661.

61. Ibidem p. 197. Trad. fr., op. cit. p. 663.

62. Ibid. p. 195. Trad. fr., op. cit. p. 661.

63. Cf. supra.

64. „Ich glaube wirklich an etwas, und das nenne ich 'ein Tag wird kommen' [...] Wahrscheinlich wird es nicht kommen [...] und trotzdem glaube ich daran. Denn wenn ich nicht mehr daran glaube, kann ich auch nicht mehr schreiben. “ dans I. Bachmann, GuI, op. cit., p. 145.

65. Dans la cinquième Leçon de Francfort qui traite de la littérature comme utopie, I. Bachmann oppose à la « langue médiocre » („die schlechte Sprache“) de la vie, une utopie de la langue („ein Utopia der Sprache") dans V. Frankfurter Vorlesung, op. cit., p. 268. Le travail sur la langue est aussi un apprentissage du silence, plus exactement, la tentative de montrer avec les mots ce qu'ils ne sont pas en mesure de dire : „ich kann redend nur mit der Ohnmacht der Rede auf etwas hindeuten, das mir wichtiger erscheint als das idiotische Gerede von [der] Rolle des Schrifstellers gestern, heute und morgen." (W. 4 p. 297).

66. Cf. A. Gargani, L'Étonnement et le hasard, Chemin de ronde et Éditions de l'Éclat, traduit de l'italien par J.-P. Cometti et J. Hansen, 1988, p. 62.

67. Ibid., p. 63.

\section{RÉSUMÉS}

À partir du récit de Ingeborg Bachmann Undine geht, nous nous attacherons à faire ressortir le mouvement croisé entre le cri de Undine d'une part, entendu comme expression de sa solitude, de son impuissance mais aussi de sa volonté de dire non à la raison raisonnante et calculatrice, non au « langage des escrocs » et, d'autre part, son appel, entendu comme chant de sirène, par la force duquel l'art et la littérature cherchent à retenir le navigateur de passage dans un instant d'éternité et de vérité, à l'arracher au temps et à la mort. Mais de l'eau mouvante du fleuve à la terre ferme, la rive, toujours, sépare, trace une frontière. La halte sur la clairière ne se retient que dans le souvenir, le récit qui se nourrissait de sa clarté fait place à une rhapsodie syncopée, à un cri véhément qui proteste contre l'oubli et la trahison, tout en s'obstinant à faire signe vers une utopie. «Un jour viendra » dit I. Bachmann, Undine le croit également et en assume le prix : un chemin épineux et douloureux, une dérive vers l'utopie. 
Von der Erzählung Ingeborg Bachmanns Undine geht ausgehend, wollen wir die wechselnde Perspektive hervorheben zwischen Undines Schrei als Ausdruck ihrer Verlassenheit einerseits, ihrer Ohnmacht aber auch ihres Willens, sich nicht auf die „vernünftelnde“, zweckmäßig berechnende Vernunft, auf die „Gaunersprache“ einzulassen, und Undines Ruf andererseits, jenem Sirenengesang, aus dem Kunst und Literatur ihre Macht schöpfen, den vorbeifahrenden Seefahrer einen Augenblick lang in der Zeitlosigkeit und in der Wahrheit aufzuhalten, ihn der Zeitlichkeit und dem Tod zu entreißen. Aber immer trennt das Ufer das wogende Wasser des Flusses vom Festland und zieht eine Grenze. Der Aufenthalt auf der Lichtung lebt nur in der Erinnerung fort, die Erzählung, die sich aus deren Licht nährte, weicht einer Rhapsodie mit synkopischer Rhythmik, einem ungestümen Protestschrei gegen Vergeßlichkeit und Verrat, und weist dabei dennoch hartnäckig auf einen utopischen Zustand hin. „Ein Tag wird kommen“ sagt I. Bachmann, Undine will es auch glauben und zahlt freiwillig den Preis dafür: einen leidvollen Dornenweg, ein schmerzliches Abtreiben auf eine Utopie hin.

Taking Ingeborg Bachmann's story, Undine geht, as a starting point, we attempt here to illustrate the chiasmus made between Undine's cry, on the one hand, understood as the expression not only of her loneliness and helplessness but also of her desire to reject rationality and calculation, and the "language of crooks"; and, on the other hand, her call, which is understood as a siren's song, the power of which is used by art and literature to arrest the passing sailor in a moment of eternity and truth, to rescue him from time and death. But the riverbank always creates a separation, and marks a boundary between flowing water and dry land. The pause in the clearing is but a memory, the story that drew from its illumination gives way to a syncopated rhapsody, a vehement cry which protests against oblivion and betrayal, while persisting in summoning up utopia. "The day will come» I. Bachman says. Undine also believes this, and accepts the consequences: a winding and painful path, a drift towards utopia.

\section{AUTEUR}

\section{MARTINE SFORZIN}

Université de Valenciennes et du Hainaut-Cambrésis 